\title{
21
}

\section{How to improve company performances from outside: a benchmarking model}

\author{
Mario Lucertinia, Fernando Nicolòb, Daniela Telmonc \\ aCentro Volterra, Università di Roma Tor Vergata,via della Ricerca Scientifica,00133-Roma (I) \\ bipartimento di Meccanica e Automatica,Università di Roma III,via Segre 2, 00153-Roma (I) \\ cTradeoff, servizi e consulenza per le aziende, vicolo del Cedro 3B, 00153-Roma (Italy)
}

\begin{abstract}
Benchmarking is an approach used for evaluating and improving company performances, by comparing them to the best performing companies. Benchmarking first studies the process to be improved, evaluate the performances, finds a best practice process in order to try to match two parts of the processes which have analogies, and then tries to change or modify the interconnections, structures or behaviour of the part to be improved using the analogy with the best trasformation process, to transform performance evaluation into improvement decisions. In the paper, we try to define benchmarking from a modelling point of view, on the ground of a suitable representation of the production system.
\end{abstract}

\section{INTRODUCTION}

Many authors conducted extensive investigations on managerial behaviour, in particular on managers who make decisions with incomplete information. Most non programmed decisions involve too many variables for a thorough examination of each and managers rarely consider all possible alternatives for the solution of a problem. Instead of attempting to maximize, the modern manager satisfices; he examines the five or six most likely alternatives and makes a choice from among them, rather than investing the time necessary to examine thoroughly all possible alternatives.

In fact, many of the so called non programmed decisions contain too many variables to be examined one by one. Thus, in a bounded rationality context, there exists, in every problem situation, a series of boundaries or limits that necessarily restrict the manager's picture of the world. Such boundaries include individual limits to any manager's knowledge of all the alternatives as well as such elements as policies, costs and technology that cannot be changed by the decision maker. As a result, the manager seldom seeks the optimum solution but realistically attempts to reach a satisfactory solution to the problem at hand.

From a modelling point of view, the boundaries include both decision variables and constraints: the decisions are taken on a subspace of the real decision space and on a subset of the complete set of the feasible decisions in the subspace. The main element conditioning decisions are resources.

By definition, anything that can be used to help solving a problem is a resource. Resources include time, money, personnel, expertise, energy, equipment, raw materials, and information.

Contraints are factors that impede problem solving or limit managers in their efforts to solve a problem. Lack of adeguate resources might prove to be a significant constraint.

Other element such as worker attitudes or government programs may prove to be a resource, a constraint, or both.

In decision making studies, many reserchers have concentrated on the analysis of alternatives with given constraints. 
Limited attention has up to now received constraints analysis.

On the other hand, constraints seem to be the real object of benchmarking and the most important constraints that can be used as drivers of an improvement process depend on the embedding of a set of interconnected activities or operations in a set of interdependent organizational units $(o u)$.

Different authors have developed typologies of organization structures, with boundaries and constraints. There are, first of all, contraints on resources. These may (at a certain cost) be or may not be removed. Non removable contraints are usually physical, technological or environmental constraints. There are contraints on the flows of materials, that can be in most cases modified only with regard to constraints linked to wastes. There are logical constraints, such as precedence and concurrency, that in many cases can be removed by a different assignment of operations to $o u$, although with an additional cost. In fact, an important subset of logical constraints are organizational contraints, that can be often modified by suitably modifying the links among ou, e.g. company procedures.

Benchmarking is a way to face the problem of modifying the organization structure and the operations assignment in a rational way. It is often defined as:

continuing search, measurement and comparison of products, processes, services, procedures, ways to operate, best practices that other companies have developed to obtain an output and global performances, with the aim of improving the company performances.

This definition can be endorsed, but, to be more specific, it is important to focus the attention on structural changes in the organizations.

In fact, the performance optimization on the ground of a given structure and/or structural changes obtained with a sequence of small local changes, are classical way to operate, coherent with the incremental culture of engineering and management.

In a rapid changing environment such changes do not fit, in many cases, the competitiveness goal; we need more effective tools.

The problem is that major modifications, even if theoretically sound, could prove to be inpractical. To convince management that relevant structural changes could be done, we must give evidence that the new organization could work. A widely recognized good proof is that others companies have adopted with success the new organization.

This paper, although a simple work in progress without numerical applications, tries to formalize a benchmarking model, which could lead to an improvement metodology.

The performance indicators are introduced in this framework, not only for comparaison purposes, but also to find guidelines for improvement.

\section{THE PRODUCTION SYSTEM: THE ACTIVITIES, THE ORGANIZATION AND THE DECISION PROCESS}

The behavior of a production system is driven by three main elements: activities, organization, and assignment of activities to organizational units $(\mathrm{ou})$.

An activity is any action aimed at trasformation of materials and/or information, including an activity aimed at the production of a decision. The activities are linked one to the other and they determine the flows of materials and/or informations throughout the system. Following a standard classification of activities, we can divide them in three main categories: production, support and management. Production activities concern products, i.e. the flow of materials/informations/money, which are the primary goal of the production system. Support activities concern all the facilities and informations needed for production and management. Management activities concern the decisions on the production flows, support and interconnection functions, all necessary for the system to function. Following this classification, a subset of the resources needed for the production belongs to the production 
flows, the remaining part belongs to the support flows. A transformation process is a set of interconnected activities.

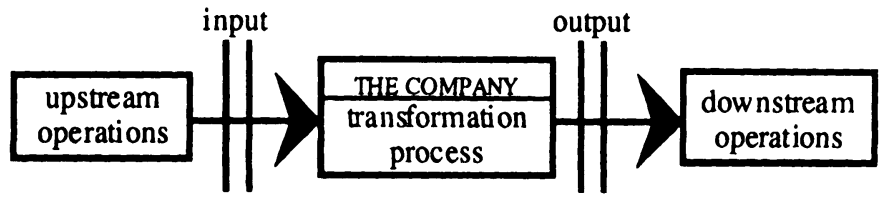

The organization concerns the choice, in a given set of feasible possibilities, of the set of resources the company is willing to use for production, the clustering of the resources into ou, and the way these ou interact one with the other, exchanging informations and materials, through pratices and procedures. Notice that resources are considered both in the flow representation of the production system and in the organization. In fact, a resource, e.g. a machine, belongs to a ou and is managed there, but its use becomes part of the production operations and therefore is included in the flows of activities. The ways to use a machine for a given set of activities are part of the activity management introduced above, the decisions concerning new machines or relevant modifications of the machine configuration or interconnection with other machines are part of the organization.

The assignment of activities to ou concerns the embedding of a production in an organization structure, so as to obtain a feasible system and a good performance. This is a standard task of the company top and middle management: who is in charge of what has to be done in each time interval and who is performing the job.

Following this representation,there are apparently two ways of looking at the system. The first begins with the existing organization and finds the best fit of activities onto the given organization, eventually with minor ex post adjustments. The second way, is to begin with the activities to be performed, and try to build around them the best suitable organization. This often drives the need of new activities to implement the new organization. These activities are difficult to foresee a priori and, in particular, it is difficult to determine before hand the quantity and quality of resources needed. This has serious effects on the performances and produces often significant delays in time.

One of the contributions of the the total quality mouvement has been that of understanding that the best solution can be obtained by looking at the system in an integrated way, together with a continuous redefinition of the company's resources involved in the organization, in a continuous improvement approach.

In this new approach, a process, traditionally seen as a set of activities and flows, is now seen as a set of activities and flows embedded in an evolving organization structure.

Most of the performance indicators concerning money, materials and informations (and the whole control activity) have been traditionally based only on the production flows. Even the total quality movement still has difficulties in creating indicators for support and managerial flows dimension on one side, and on the organizational dimension on the other side.

The field measures are taken in particular points of the system, suitably related to the transformation process considered (see the above flow chart describing the production flow), and are in terms of quantities (flows, levels and time). On the other hand, performance indicators are taken at different times or time intervals, and adeguately elaborated. Typical performance criteria are: effectiveness, efficiency, productivity, profitability (or budgetability), quality. 
On the other hand, examples of integration indicators, taking into account the organization structure and the embedding of the activities, are indicators of flexibility (range, uniformity, mobility,...) and complexity (connectiveness, procedures path lenght, number of ou interconnections,...).

In order to determine what and where to measure, and which are the right performance indicators and how they relate to measurements, we must follow two basic directions: internal relevance and external consistency.

As far as internal relevance is considered, it is important to define company goals and constraints. To put together goals, measures and performances, you need a conceptual model of the transformation process, that can be used to transform performance evaluation into improvement decisions.

As far as external consistency is concerned, it is important that all indicators will be the same in all the companies, and therefore they will be comparable.

The decision making process, its link with the value of a set of performance indicators, suitably depicting the company's behaviour, and the organization supporting the process, are a cornerstone of the benchmarking building.

In practice, company decisions lie on different levels. From the viewpoint of this paper we can introduce three levels of decisions: strategic, tactical and operational. Using decision models' language, we may characterize the three levels as follows:

\section{Operational level}

Given: environment, operational conditions and procedures, information flows, operational constraints (different types of technological and organizational contraints), a univocally defined objective function,...;

find: the value of decision variables directly connected to the process;

such that: the performance will be optimized (throughput maximization, lead time minimization, ecc.).

\section{Tactical level}

Given: environment, structural constraints difficult to modify, the company goals, a set of performance indicators;

find: operational constraints, information flows, operational procedures and the value of decision variables;

such that: the organization performances will be optimized (flexibility, complexity, ecc.).

\section{Strategic level}

Given: environment, global structural constraints and resources, set of interconnected decision centers;

find: company goals and performance indicators;

such that: the profitability of investments will be maximized.

Benchmarking focuses only on certain types of intermediate decisions, that we have called of tactical level.

These decisions do not concern, typically, basic company strategies, such as market selection, process selection, joint ventures. In the following, to focus the attention on the intermediate level, we will suppose that strategic decisions have been already taken and cannot be modified.

In the same way, benchmarking decisions do not concern operational decisions, such as material routing and operations scheduling. We will suppose that, given the set of constraints, internal efficiency (i.e. a good solution) is always achieved by the decision makers at the operational level. All actions at this level can therefore be considered completely determined by the upstream decisions and the environment. For instance, we may suppose that, in the constrained optimization of the operational level, where all actions are performed on the ground of the choices made at the tactical level, the decision process can be represented in an 
optimization format with a unique decision maker and complete information, where the output of the tactical level produces the constraints of the optimization problem.

To simplify the notation, in the next section the optimization model of the operational level is assumed to be in a linear programming format.

Benchmarking decisions focus on a tactical level, where you can modify organizational constraints, procedures and practices. We have had examples of this in physical material handling, distribution systems, assembly lines, production layout, make or buy, precedence or concurrency constraints (a usual way to represent the relations introduced by the information flow through the organizational units of the system).

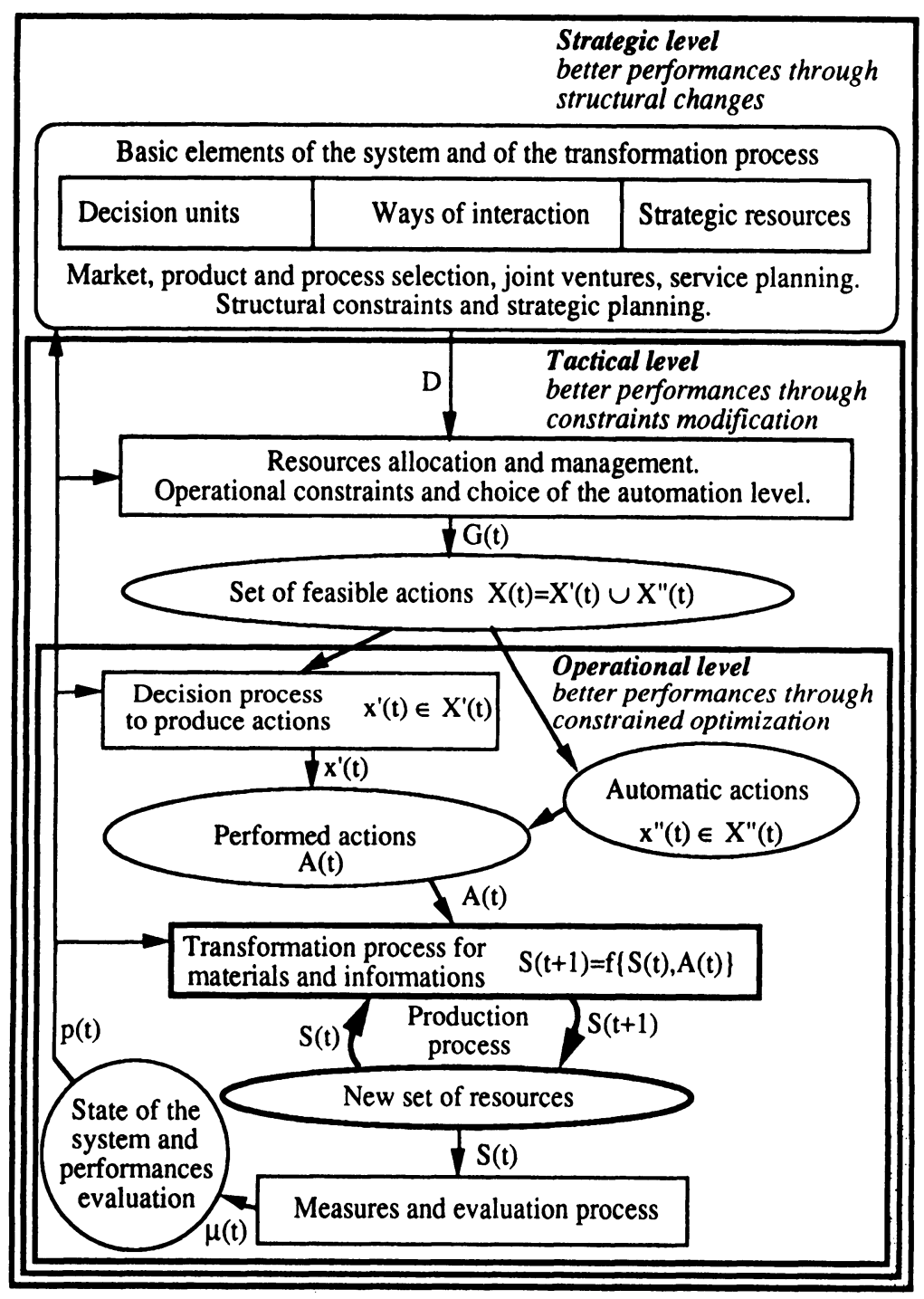


Traditionally, in managing a production system, you generally start by defining the activities of all the organizational units, then the interconnection network that links them, taking only implicitly into account the support services' need. Starting from this breakdown into units' activities, you aggregate the units on the basis of the interaction network in order to define aggregated processes and their coordination needs. But in this way the breakdown becomes an organizational constraint, and the performance optimization process is typically done in the framework of a given organization structure.

On the other side, organization structure is often the main obstacle to improvement, and the optimization of such a structure is a problem much too wide and undefined.

Benchmarking, as it will be better explained in the next sections, is a way to handle this problem. The activity breakdown is performed on the ground of a set of different organization structures and for each of the resulting systems, the performances can be evaluated. Instead of looking among all possible organization structures, we limit our search to existing ones. To make this effective, you must be able to compare not only similar companies, but also very different ones, and therefore we must work on subsystems that have the same input-output functional model. The subsystems to be compared are found bottom up, aggregating the unit activities on the ground of the relationship among units. The breakdown is performed on the process implemented by the two subsystems.

We may therefore say that we compare two companies where the same function is performed in two different ways, corresponding to two different breakdowns (i.e. two different organizations and/or two different assignments). These breakdowns are, in our opinion, one of the main aspects of benchmarking.

In order to make an activity breakdown, it is important to describe, the functional relations among input, transformation activities and output, the resources consumed and the resources available for the activities, the aggregation of resources into $o u$, the links among ou and set of ou. Given the resource/activity connections and the relations amongst activities, we can define the resource allocation process and plan our activities in time.

\section{THE OPERATIONS BREAKDOWN TREE}

Let $\mathrm{P}$ be a given process which transforms, in a suitable time interval, a given input in a given output. $P$ is composed by a set of interdependent operations. Let $U$ and $U^{p}$ be the sets of $o u$ in charge of performing the process $P$ in company $C$ and in a partner company $C^{p}$. In fact, for benchmarking, we assume the point of view of company $C$ and we will use company $C^{p}$ as the benchmarking partner; as already said, benchmarking is not a redesign from scratch of a process, but a redesign based on the transfer of processes existing in other companies.

Several structured metodologies and tools developed for organizational analysis may be of help to us for finding technological coefficients for resource constraints and for expressing performance indicators, usually defined in terms of products and final outputs, as a function of the activities in which the process breaks down and of the ou in which the company resources are partitioned. To do this we need to associate products to activities and activities to resource consumption.

The standard breakdown of activities required by the benchmarking analysis produces a decomposition useful for comparing processes in different companies and may be used for finding standards of performance for some types of processes.

In developing the activity breakdown, some subsets of activities corresponds to well defined $o u$, with formalized interactions with the other ou and with the external organizations, other activities cannot be properly embedded in the process' ou and must therefore be considered separately. In the following, these activities are considered part of the support. In general, support activities are only partially devoted to the process we are considering, but they are also included in different external processes. 
The hierarchical breakdown produces a breakdown tree. Each breakdown of a node of the tree produces a set of nodes corresponding to a partition of the activities of the transformation process and, in case, two additional nodes: one for all support needs not included in the transformation process considered above (e.g. maintenance, purchasing, delivery, administration,...), another for the interconnection among set of activities belonging to different elements of the partiton and the interconnection between them and the outside environment (e.g. transportation systems, information network, rules and procedures,...). In the following, we will indicate the nodes of the tree as process, support and network nodes. Process nodes correspond to all the different phases of the transformation process at different levels of aggregation. Support nodes enable process nodes to operate effectively. Network nodes correspond to connection and integration procedures among all its brothers; more precisely, each network node induces an interaction graph among a set of process nodes (e.g. precedence constraints, information flows,...). Support and network nodes have no sons and represent part of the organization structure.

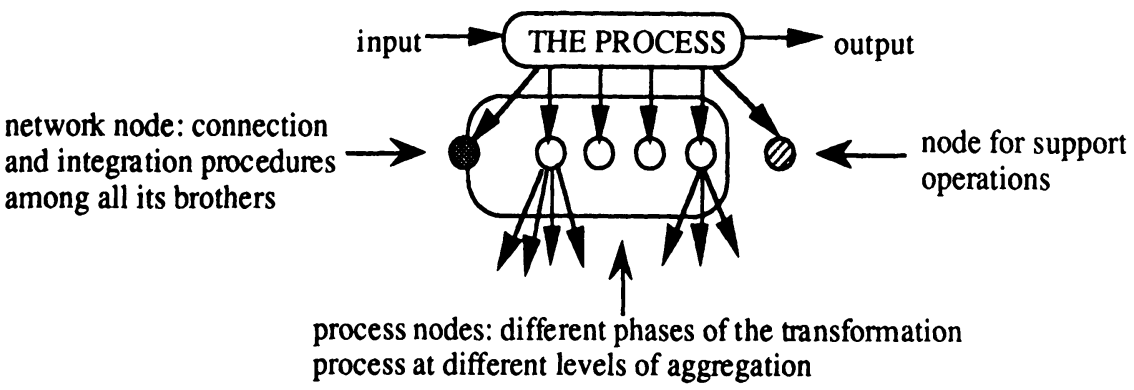

The breakdown's first level of decomposition

Each leaf of the tree corresponds to a set of activities which are assigned to a unique ou; for process nodes, each ou is typically in charge of only one leaf. This is often the case when the organization has been designed ad hoc for the process.

Let $\mathrm{T}$ and $\mathrm{T}^{\mathrm{p}}$ be two operations breakdown trees $(o b t)$ of $\mathrm{P}$.

In general, $\mathrm{T}$ and $\mathrm{T}^{\mathrm{p}}$ will have several subsets of equivalent nodes. By equivalent we mean that input and output (therefore the transformation process) are the same, although the transformation may be obtained with a different set of operations, different decision variables and using a different set of resources.

Notice that, if we consider a transformation process of company $C^{p}$ (node of $T^{p}$ ) and we try to transfer this process to company $\mathrm{C}$ substituting it to the corresponding node, we must anyway redesign the process and adapt it to the caracteristics and features of company $\mathrm{C}$. When we redesign the process, we may either find that the transformation is the same as the original one in company $\mathrm{C}$, or that it is different. In the first case the two nodes are called identical. In the second case an opportunity for benckmarking becomes possible. Two equivalent nodes can be both leaves, both internal nodes, one leave and one internal node.

If a node of a tree has no equivalent node in the other tree, then it may belong to a subtree which have a corresponding alternative subtree in the other tree; the roots of the two subtrees are equivalent nodes, although not identical. In this case a benckmarking opportunity becomes possible, by replacing the whole subtree.

Every time that we find the opportunity of substituting a node or a subtree of $\mathrm{C}$ with a node or a subtree of $\mathrm{C}^{\mathbf{p}}$, we introduce an or node representing this choice.

Let $\mathrm{T}^{\mathrm{aO}}$ be a tree with and-or branches, obtained as the union of $\mathrm{T}$ and $\mathrm{T}^{\mathrm{P}}$ (always from the point of view of company $C$ ). The set of or nodes represents the set of choices available for the benchmarking. Each or is the root of two subtrees (that may also be a single node, the left 
subtree is a subtree of $T$, the right subtree is a subtree of $T^{p}$ ) which perform the same inputoutput functions (although in a different way, with a different set of decision variables and using a different set of resources).

and-or branches are obtained by integrating in the breakdown tree the benchmarking partner's processes, ways to operate and best practices

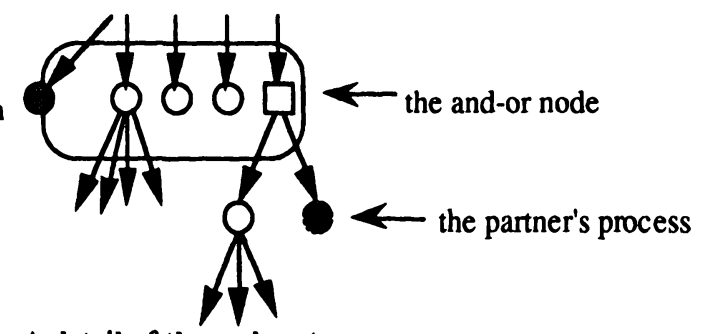

A detail of the and-or tree

Even though, in principle, all combinations are possible (with a different set of constraints), to better clarify the benchmarking process, we may consider separately meanigful aspects.

The most important aspect is organizational benchmarking, which studies the possibility of substituting only subtrees corresponding to different breakdowns of subactivities. In this case we suppose that company $\mathrm{C}$ has designed at best the elementary tranformation processes, their interconnection and the support; the only constraint in obtaining better performances is a not optimal breakdown.

A second aspect is integration benchmarking, which studies the possibility of substituting only network nodes. This means either a different set of interconnections, or a different way to manage them.

A third aspect is implementation benchmarking, which studies the possibility of substituting only single process and support nodes (supposing therefore that the interconnections and the nodes are the same in the two trees). In this case we suppose that the design of some single units may be improved.

A fourth aspect is goal benchmarking, which studies the possibility, for two trees with all identical nodes and a benchmark expressed in terms of a set of values of performance indicators, that the actions of company $\mathrm{C}$ does not fit the benchmark.

\section{A BENCHMARKING MODEL}

The decisions associated to the operation breakdown tree $T$ introduced in the last paragraph, produce actions given in term of a vector of variables $x \in X$ (where $X$ is the decision space of company $C$ for process $P$ ). Each entry of $x$ belongs to a unique leaf of $T$. It may be a continuous variable (tipically associated to process leaf) or a binary variable (tipically associated to a network node, where it represents a link). Variables associated to identical nodes belong to the same decision subspace.

The constraints of company $C$ (in a linear programming format) can be written as $A x \leq b$, where $A$ is a generalized technology matrix and $b$ is a generalized resource vector. The value of the entries of $A$ results from the design of the units and the network. In our model, we assume that the value $b$ cannot be modified by benchmarking; this means, in particular, that $b$ must support both the activities of $T$ and the activities of $T^{p}$ redesigned for $C$.

The objective function of company $C$ (to be maximized) can be written as $f(x)$ and can often be expressed as a linear combination of performance indicators. We can suppose that performance indicators can be expressed as function of the decision variables that drive the process. In fact, such variables, together with the initial state of the system, determine the state in all the following time frames (because of the assumption of a deterministic system), the state of the system determines the output and the output determines the performances. When, in 
order to find better performances, we incorporate some processes of another company into our company, the decision variables can change, but the performance indicators remain the same (even though, hopefully, with a better numerical value).

Let us indicate as $I(x)$ the performance indicators vector function of a given decision vector $x$ and $I^{*}$ the best performance vector, obtained examining the performances of the best companies (competitors or companies performing that function better then the others).

The whole operational level decision model can therefore be written as:

(OL)

$$
\max f(x)
$$

$x \in X$

$\mathrm{Ax} \leq \mathrm{b}$

In the following we will present a model based on organizational and goal benchmarking. The analysis can be easily extended to the other two aspects mentioned above. We therefore assume that in $\mathrm{T}^{\mathrm{aO}}$ there are no or nodes between single nodes (i.e. all the single leaves are designed at best) and $\mathbf{k}$ or nodes between two subtrees with different breakdowns (i.e. for each path from the root to a leaf there is at most one or node).

The colums of matrix $\mathrm{A}$ and vector $\mathrm{x}$ are divided into blocks: one block for all the variables belonging to the leaves corresponding to identical nodes in $\mathrm{T}_{\text {and }} \mathrm{T}^{\mathrm{P}}$; one block for each or node, corresponding to the variables belonging to the leaves of the left subtree of the or node (the subtree belonging to $\mathrm{T}$ ).

$$
\begin{gathered}
A=\left[A_{0}\left|A_{1}\right| A_{2}|\ldots| A_{k}\right] \\
x^{T}=\left[x_{0}{ }^{T}\left|x_{1}{ }^{T}\right| x_{2}{ }^{T}|\ldots| x_{k}{ }^{T}\right]
\end{gathered}
$$

The bloks from 1 to $\mathrm{k}$ can be replaced by a suitable redesign of the corresponding processes of the company $\mathrm{C}^{\mathrm{p}}$. Let us indicate such blocks and the correponding variables (assumed to belong to a given decision space $\mathrm{Y}$ ) as:

$$
\begin{gathered}
R=\left[R_{1}\left|R_{2}\right| \ldots \mid R_{k}\right] \\
y^{T}=\left[y_{1}{ }^{T}\left|y_{2}{ }^{T}\right| \ldots \mid y_{k}{ }^{T}\right]
\end{gathered}
$$

Company $\mathrm{C}$ verifies that its performance is lower then the market needs, decides that the performance benchmark in given by I* and tries to reach such performance by introducing a suitable subset of the $k$ alternative processes of $C^{p}$. Let $B$ be a $0-1 k$-vector, each value of $\beta$ corresponds to one of the $2^{k}$ subsets of activities belonging to $C^{p}\left(B_{i}=0\right.$ if the $T$ activities is taken, $B_{i}=1$ if the $T^{p}$ activities is taken). In practice $k$ is relatively small and all possible combinations can be easily analized.

Let $\mathrm{z}$ be a decision variables vector obtained by chosing the decision variables $\mathrm{x}$ or $\mathrm{y}$ depending on the value of $B$ and $Z$ the correspondent decision space:

$$
\mathrm{z}^{\mathrm{T}}=\left[\mathrm{z}_{0} \mathrm{~T}^{\mathrm{T}}\left|\mathrm{z}_{1}{ }^{\mathrm{T}}\right| \mathrm{z}_{2}{ }^{\mathrm{T}}|\ldots| \mathrm{z}_{\mathrm{k}}{ }^{\mathrm{T}}\right]=\left[\mathrm{x}_{0}{ }^{\mathrm{T}} \mid \mathrm{x}_{1}{ }^{\mathrm{T}} \text { or } \mathrm{y}_{1} \mathrm{y}_{1}{ }^{\mathrm{T}} \mid \mathrm{x}_{2}{ }^{\mathrm{T}} \text { or }{ }_{2} \mathrm{y}_{2}{ }^{\mathrm{T}}|\ldots| \mathrm{x}_{\mathbf{k}}{ }^{\mathrm{T}} \text { or }{ }_{k} \mathrm{y}_{\mathrm{k}}{ }^{\mathrm{T}}\right]
$$

where $o r_{i}$ chose $x_{i}$ if $\beta_{i}=0$, chose $y_{i}$ if $\beta_{i}=1$. In the same way we can obtain a new matrix $D$.

$$
\mathrm{D}=\left[\begin{array}{l}
\mathrm{D}_{0}\left|\mathrm{D}_{1}\right| \mathrm{D}_{2}|\ldots| \mathrm{D}_{\mathrm{k}}
\end{array}\right]=\left[\begin{array}{llll}
\mathrm{A}_{0} \mid \mathrm{A}_{1} \text { or } & \mathrm{R}_{1} \mid \mathrm{A}_{2} \text { or } & \mathrm{R}_{2}|\ldots| \mathrm{A}_{\mathrm{k}} \text { or } \\
\mathrm{R}_{\mathrm{k}}
\end{array}\right]
$$

Notice that the number of entries of $z$ is equal to the number of columns of $D$.

As the performance indicators are function of the decision variables, when we introduce activities of company $C^{p}$ into company $C$, they become functions of the new decision variables. 
We will indicate as $I(z)$ the indicators vector obtained by chosing the decision variables $x$ or $y$ depending on the value of $B$.

The organizational benchmarking model can be written as:

Model BM(B)

$\begin{array}{lll}\text { Given: } & A, R, I^{*} & \\ \text { Find: } & z & \text { (i.e. } x, y) \\ \text { Such that: } & D(B) z \leq b & \left.\text { (i.e. } A_{0} x_{0}+\sum_{i=1, \ldots, k}\left\{\left(1-B_{i}\right) A_{i} x_{i}+B_{i} R_{i} y_{i}\right\} \leq b\right) \\ & z \in Z(B) & \text { (i.e. } x \in X \text { and } y \in Y) \\ & \partial\left[I^{*}-I(z)\right]^{+} \text {is minimum }\end{array}$

where $\partial[\cdot]^{+}$is a weighted sum of the positive values of the argument entries.

In practice $B M(B)$ is a goal programming problem.

Notice that, if $I(z)$ can be approximated by a linear function and there are no binary variables, we can apply the results of Data Envelopment Analysis [ccgss] in order to find the optimal value of $B$.

If $B=0$ we have an example of what we have indicated in the former section as goal benchmarking. In fact, if $\mathrm{BM}(0)$ has a solution with $\partial=0$ (i.e. $\left.\mathrm{I}(\mathrm{z}) \geq \mathrm{I}^{*}\right)$, then $\mathrm{C}$ has the possibility of reaching the benchmark I* without structural changes, but the company goal $f(x)$ pushes the company in a wrong direction. We must therefore change the company objectives, and consequently change the activities, to reach the goal.

\section{CONCLUSIONS}

The paper presents a modelling approach to benchmarking. The paper tries to produce a structured methodology for benchmarking: four types of benchmarking are put into evidence.

One type of benchmarking is goal benchmarking, which studies the possibility, based on the improvement of performance indicators, of trying to get the values of the benchmark.

Even more important, according to our opinion, is organizational benchmarking, as we have called it, which studies the possibility of substituting sets of activities of the whole process with other sets of the breakdown activities of the same process in the best practice company.

Another aspect is integration benchmarking, which studies the possibility of changing the interconnection pattern for the same activity breakdown (this case is fairly rare on its own, because, in general, only few interconnections are possible).

The last aspect is implementation benchmarking, which studies the possibility of redesigning process or support units. In this case we suppose that the design of some single units may be improved.

An optimization model, based on a linear programming format, has been introduced, to exemplify the approach, combining the goal and the organizational benchmarking.

From an algoritmic point of view, the solution of the benchmarking problem formulated here is related, for linear indicators, to data envelopment analysis.

The paper tries to give a frame for benchmarking analysis; a future aim is to validate the approach in a few meaningful cases. This validation activity is now in progress in two different fields: information systems for manufacturing and information systems for university services.

\section{REFERENCES}

[bon] C.Bonini, Simulation of information and decision systems in the firm. Prentice-Hall, 1963.

[cam] Robert C.Camp, Benchmarking. the search for industry best practices that lead to superior performance. ASQC quality pres, 1989. 
[ccgss] A.Charnes, W.W.Cooper, B.Golany, L.Seiford, J.Stutz, Foundation of Data Envelopment Analysis for Pareto-Koopmans efficient empirical production functions, J. of Econometrics, vol.30, n.1/2, 1985, pp.91-107.

[hc] Micheal Hammer, James Champy, Reingineering the corporation, Hamper business, 1993.

[hub] G.Huber, $A$ theory of the effects of advanced information technologies on organizational design, intelligence, and decision making. Academy of Management Review, 15, 1, pp.47-71, 1990.

[jk] H.Thomas Johnson, R.S.Kaplan, Relevance Lost: the rise and fall of management accounting, Boston: Harvard Business School Press, 1987.

[kap] Robert S.Kaplan (ed.), Measures for manufacturing excellence, Harvard Business School Series in Accounting and Control, 1990.

[kp] A.Kumar, P.S.Ow, M.J.Prietula, Organizational simulation and information systems design: an operations level example. Management Science, 39, 2, pp.218-240, 1993.

[lc1] Byron C.Lewis, Albert E.Crews, The Evolution of Benchmarking as a Computer Performance Evaluation Technique, MIS Quaterly, 9, $\mathrm{n}^{\circ} 1$ (march 1985), pagg.8-16.

[lc2] Richard L.Lynch, Kelvin F.Cross, Measure up: yardsticks for continuous improvement. Basil Blackwell inc., 1991.

[In] Kathleen H.J.Liebfried, C.J.McNair, Benchmarking: a tool for continuous improvement, Harper Business, 1992.

[lnc] Mario Lucertini, Fernando Nicold, Daniela Telmon, Integration of benchmarking and benchmarking of integration, Nato Advanced School on Integration, Il Ciocco, Italy, 1993 (to appear on Int.J.on Production Economics).

[mas] Brian Maskell, Performance Measurement for World Class Manufacturing: a Model for american companies, Productivity Press, 1992.

[ms] T.Malone, S.Smith, Modelling the performance of organizational structures. Operations Research, 36, 3, pp.421-436, 1988.

[mt] Kathleen Malette, Joanne Tomlinson, Benchmarking: focus on world class practices, AT\&T Bell Laboratories Technical publication Center, 1992.

[saa] Thomas L.Saaty, Decision making for leaders: the analytical herarchy process for decisions in a complex world, RWS Publications, 1988.

[spe] Micheal J.Spendolini, The benchmarking book, AMACOM, 1992.

[tzc] Fancis G.Tucker, Seymour M.Zivan e R.C.Camp, How to measure Yourself against the best, Harvard Business Review, jan.feb. 1987, pagg.2-4.

[wat] Gregory H.Watson, The benchmarking workbook: adapting best practices for performance improvement, Productivity Press, 1992.

[you] Edward Yourdon, Modern structured analysis, Prentice-Hall, 1989. 